\title{
Editorial
}

Nephrology

Published online: September 25, 2019

DOI: $10.1159 / 000502899$

\section{Kidney Injury Biomarkers with Clinical Utility: Has Godot Finally Arrived?}

\author{
Steven G. Coca \\ Division of Nephrology, Department of Medicine, Icahn School of Medicine at Mount Sinai, New York, NY, USA
}

The search for relevant kidney injury biomarkers has been a fervent one and well-documented over the past 15 years. Since the "Big Bang" of the kidney injury biomarker era, demarcated by the seminal study by Mishra and colleagues in which urine neutrophil gelatinase-associated lipocalin (NGAL) showed almost perfect (i.e., too good) performance for the early diagnosis of acute kidney injury (AKI) [1], there have been over 1,000 papers on urine biomarkers in AKI and another $700+$ papers on urine biomarkers for outpatients at risk for incident or progressive chronic kidney disease (CKD). The 10th Acute Dialysis Quality Initiative meeting disseminated a report and recommendations 5 years ago that aimed to "equip physicians to effectively use biomarkers in AKI" [2]. The recommendations proposed the use of combinations of functional and damage biomarkers to "facilitate determination of prognosis and ultimately lead to improved outcomes". In the same year, the Food and Drug Administration approved NephroCheck ${ }^{\circledR}$ to provide a risk assessment for moderate or severe AKI within $12 \mathrm{~h}$ of assessment in critically ill patients.

Where have all these publications and Food and Drug Administration approvals on kidney injury biomarkers gotten us clinically? Have they resulted in changes in the incidence or outcomes related to AKI? Data from Ireland demonstrated a doubling of the AKI incidence from 2005 to 2014 [3]. Data from England showed a much higher increase (nearly tenfold) between 1998 and 2013 [4]. Studies from the United States and the United Kingdom showed similarly sobering trends in AKI requiring dialysis [5-7]. Clearly, there is no evidence to date that the explosion of research funds, time, and resources devoted to kidney injury biomarkers in the recent years has translated into improvements in kidney-related clinical outcomes. Part of the reason may be that uptake and clinical usage of these biomarkers has in general been low, in the United States and outside the United States.

The second issue may relate to the syndrome of AKI itself in hospitalized patients. It is a complex syndrome that often results from multiple different insults, and there is no specific prevention or treatment strategy that can be employed. Even when tested in a randomized controlled trial, randomization of patients who underwent cardiac surgery and had high levels of NephroCheck ${ }^{\circledR}$ post-operatively to the Kidney Disease Improving Global Outcomes "Kidney Disease Improving Global Outcomes bundle" (consisting of optimization of volume status and hemodynamics, avoidance of nephrotoxic drugs, and preventing hyperglycemia) versus standard of care reduced the incidence of post-operative AKI by serum creatinine or urine output criteria, but yet failed to translate into a reduction in any clinically meaningful outcomes at 30,60 , or 90 days [8].

Part of the issue about testing of biomarkers in the acute setting is the "so what?" question, as the treatment of AKI in hospitalized patients is completely unclear. Should patients be given more intravenous fluids or less? The reflex by most physicians is to give fluids to patients

\section{KARGER}

(c) 2019 S. Karger AG, Basel

E-Mail karger@karger.com

www.karger.com/ajn
Steven G. Coca, DO, MS

Division of Nephrology, Department of Medicine

Icahn School of Medicine at Mount Sinai

One Gustave Levy Place, Box 1243, New York, NY 10029 (USA)

E-Mail Steven.Coca@mssm.edu 
with AKI, but data repeatedly have shown that drier is better [9]. If patients have acute decompensated heart failure, should diuretics be held or be continued if kidney injury biomarkers are elevated? Counterintuitively, patients with acute decompensated heart failure who have elevated injury biomarkers and clinical AKI actually have better post-discharge outcomes and better post-discharge kidney function $[10,11]$. Should renin angiotensin aldosterone system antagonists be held or continued in patients with AKI? [12]. All of these questions are difficult, and data are sometimes conflicting or not definitive and may sometimes contradict intuition and concepts learned in training, and thus, the complexity of AKI and lack of clear treatments has almost led physicians to stick their heads in the sand on these issues.

Thus, the fact that hospital-acquired AKI is such an enigma has led to a general and slow shifting of the testing and potential application of kidney injury biomarkers from the inpatient to the outpatient setting. In this paradigm, the kidney injury biomarkers, if they reach the clinical arena, would not necessarily be used to rapidly change management or care over the ensuing few hours, but instead be used to predict long-term decline in kidney function or end-stage renal disease.

Repurposing of kidney injury biomarkers in the chronic setting still makes pathophysiologic sense, as there is presumably ongoing tubulointerstitial injury in many forms of $\mathrm{CKD}$, and in this arena, if biomarker(s) are deemed successful for prognostication, can be used to guide more informed decisions about medication regimens, referral to specialists, or delineation of higher risk patients that might be afforded benefit from relatively new, but effective therapies (e.g., SGLT2 inhibitors). Early work that examined the association of various kidney injury biomarkers (NGAL, KIM-1, NAG and L-FABP) in large cohorts of patients with [13] or without [14] prevalent CKD indeed confirmed that there was substantial elevation of these injury biomarkers in those patients that ultimately experienced progression. Enthusiasm was dampened, however, when the data also demonstrated that there was limited to no independent association between the biomarkers and kidney disease progression or end-stage renal disease after adjustment for routine clinical variables, including eGFR and urine albumin $[13,14]$.

Testing broad swaths of populations of unselected individuals with or without prevalent CKD may not be the appropriate way to study the true value of urinary biomarkers. Each marker or panel of markers will have to have demonstrated fit for purpose in groups of individuals that may have some type of unifying underlying patho- physiology of kidney disease. These groups can be potentially broken down into patients with early and/or late diabetic kidney disease, non-diabetic or APOL1-associated kidney disease, various types of glomerulonephritides, patients with chronic heart failure, chronic liver disease and HIV-positive individuals.

It is this last group of individuals (HIV positive), Drs. Jotwani, Shlipak and Ix' collaborative group has generated a modicum of data on urine biomarkers in this setting. This group previously demonstrated that in the Women's Interagency HIV Study, women with high levels of urine alpha- 1 microglobulin were at more than twofold increased risk of incident $\mathrm{CKD}$ and progressive eGFR decline, and had an adjusted $60 \%$ increased risk for death [15]. Interestingly, the associations of alpha- 1 with the kidney endpoints were as strong in women who were not infected with HIV [15]. This group of investigators also demonstrated that HIV-infected men in the Multicenter AIDS Cohort Study (MACS) had higher alpha-1 microglobulin levels than HIV-uninfected men, and that alpha-1 levels were associated with cumulative tenofovir exposure [16].

In this issue of the American Journal of Nephrology, the same group of investigators present another analysis from the MACS cohort, demonstrating that alpha-1 microglobulin (as well as urine albumin), were associated with faster eGFR decline over 4.8 years of follow-up in both the HIV+ and HIV- men. Those individuals in the highest tertile of alpha-1 microglobulin had eGFR decline of approximately $-2.5 \mathrm{~mL} / \mathrm{min} / 1.73 \mathrm{~m}^{2} /$ year faster in HIV+ and HIV- men versus those in the lowest tertile. Other urine biomarkers (IL-18, KIM-1, and PIIINP) were not associated with eGFR decline [17].

Many in the kidney injury biomarker field probably feel like Vladimir and Estragon, in Samuel Beckett's play "Waiting for Godot", who waited endlessly for ephemeral Godot, only to have the play end without ever witnessing his promised arrival. Alpha- 1 microglobulin is a $26-\mathrm{kDa}$ lipocalin that circulates in plasma in free and protein-bound forms. Unbound alpha-1 microglobulin is freely filtered at the glomerulus and reabsorbed by proximal tubular epithelial cells through the endocytic receptor megalin. When proximal tubules are damaged, alpha-1 microglobulin appears in the urine due to lack of complete reabsorption. It should be noted that alpha-1 microglobulin was also a very strong marker for predicting allograft failure in kidney transplant recipients [18]. While potentially less hyped and less "famous" than other urine biomarkers such as urine NGAL, KIM-1, IL-18, L-FABP, is alpha-1 microglobulin our Godot? Is it the 
urine biomarker that finally has clinical promise in the outpatient setting for predicting kidney function decline?

The answer is a strong maybe, but it looks like we will have to wait a little longer to see if Godot truly arrives. The first reason not to prematurely celebrate is that, in this study by Ascher et al. [19], alpha-1 microglobulin (and urine albumin) was only associated with the annual rate of eGFR decline and was not associated with the outcome of incident CKD. Second, data from the very same group of investigators using the same cohorts mentioned above (MACS and Women's Interagency HIV Study) showed that alpha- 1 could not pull off the victory for predicting changes in eGFR after the initiation of the known nephrotoxin, tenofovir, in a subset of 198 participants who initiated the drug, when compared to 13 other biomarkers in a subset of participants with HIV that were initiated on tenofovir [19]. In that analysis, other urinary biomarkers (baseline KIM-1, clusterin, and uromodulin) were associated with first year eGFR decline after the initiation of tenofovir. Alpha-1 microglobulin trended toward larger eGFR decline, but unlike KIM-1, clusterin, and uromodulin, was not statistically significant after multivariable adjustment [19].

Where does this leave us? Maybe we do not have the ideal urine biomarker ready to employ in the outpatient setting yet, "but surely tomorrow", as Vladimir and Estragon state at the end of Waiting for Godot. We will see what enlightenment we get regarding the performance and potential clinical utility of not only alpha- 1 micro- globulin but also other urinary biomarkers such as urinary EGF, MCP-1, KIM-1, and YKL-40, along with several plasma biomarkers assayed in multiple large cohorts, as part of work that will soon emerge from the CKD Biomarker Consortium (https://www.ckdbiomarkersconsortium.org/). This work will not merely have to demonstrate an "independent association" of any given biomarker or biomarkers with the kidney progression outcomes, but will have to also demonstrate some sort of clinical utility potential, in the form of strong positive or negative predictive value, before full consideration for clinical use can be justifiably entertained. Until those hurdles are crossed, I will continue to wait with Vladamir and Estragon for Godot (i.e., the urine biomarker[s] that reliably satisfies these characteristics).

\section{Acknowledgments}

Dr. Steven Coca is a member and has salary support from the CKD Biomarker Consortium (U01DK106962). He is also supported, in part, by the following grants: R01DK115562, R01HL85757, R01DK112258, and U01OH011326.

\section{Disclosure Statement}

Dr. Steven Coca is a member of the advisory board of RenalytixAI and owns equity in the same. In the past 3 years, he has received consulting fees from RenalytixAI, Goldfinch Bio, CHF Solutions, Quark Biopharma, Janssen Pharmaceuticals, Takeda Pharmaceuticals, and pulseData.

\section{References}

1 Mishra J, Dent C, Tarabishi R, Mitsnefes MM, $\mathrm{Ma} \mathrm{Q}$, Kelly C, et al. Neutrophil gelatinaseassociated lipocalin (NGAL) as a biomarker for acute renal injury after cardiac surgery. Lancet. 2005 Apr;365(9466):1231-8.

2 Murray PT, Mehta RL, Shaw A, Ronco C, Endre Z, Kellum JA, et al.; ADQI 10 workgroup. Potential use of biomarkers in acute kidney injury: report and summary of recommendations from the 10th Acute Dialysis Quality Initiative consensus conference. Kidney Int. 2014 Mar;85(3):513-21.

3 Stack AG, Li X, Kaballo M, Elsayed ME, Johnson $\mathrm{H}$, Murray PT, et al. Temporal trends in acute kidney injury across health care settings in the Irish health system: a cohort study. Nephrol Dial Transplant. 2018, Epub ahead of print.

4 Kolhe NV, Muirhead AW, Wilkes SR, Fluck RJ, Taal MW. The epidemiology of hospitalised acute kidney injury not requiring dialysis in England from 1998 to 2013: retrospective analysis of hospital episode statistics. Int J Clin Pract. 2016 Apr;70(4):330-9.

5 Brown JR, Rezaee ME, Hisey WM, Cox KC, Matheny ME, Sarnak MJ. Reduced Mortality Associated with Acute Kidney Injury Requiring Dialysis in the United States. Am J Nephrol. 2016;43(4):261-70.

6 Hsu RK, McCulloch CE, Dudley RA, Lo LJ, Hsu CY. Temporal changes in incidence of dialysis-requiring AKI. J Am Soc Nephrol. 2013 Jan;24(1):37-42.

7 Kolhe NV, Muirhead AW, Wilkes SR, Fluck RJ, Taal MW. National trends in acute kidney injury requiring dialysis in England between 1998 and 2013. Kidney Int. 2015 Nov;88(5): 1161-9.

8 Meersch M, Schmidt C, Hoffmeier A, Van Aken H, Wempe C, Gerss J, et al. Prevention of cardiac surgery-associated AKI by implementing the KDIGO guidelines in high risk patients identified by biomarkers: the PrevAKI randomized controlled trial. Intensive Care Med. 2017 Nov;43(11):1551-61.

9 Ostermann M, Straaten HM, Forni LG. Fluid overload and acute kidney injury: cause or consequence? Crit Care. 2015 Dec;19(1):443.

10 Ahmad T, Jackson K, Rao VS, Tang WH, Brisco-Bacik MA, Chen HH, et al. Worsening Renal Function in Patients With Acute Heart Failure Undergoing Aggressive Diuresis Is Not Associated With Tubular Injury. Circulation. 2018 May;137(19):2016-28.

11 Rao VS, Ahmad T, Brisco-Bacik MA, Bonventre JV, Wilson FP, Siew ED, et al. Renal Effects of Intensive Volume Removal in Heart Failure Patients With Preexisting Worsening Renal Function. Circ Heart Fail. 2019 Jun; 12(6): $\mathrm{e} 005552$.

12 Perazella MA, Coca SG. Three feasible strategies to minimize kidney injury in 'incipient AKI’. Nat Rev Nephrol. 2013 Aug;9(8):484-90. 
13 Hsu CY, Xie D, Waikar SS, Bonventre JV, Zhang X, Sabbisetti V, et al.; CRIC Study Investigators; CKD Biomarkers Consortium. Urine biomarkers of tubular injury do not improve on the clinical model predicting chronic kidney disease progression. Kidney Int. 2017 Jan;91(1): 196-203.

14 Foster MC, Coresh J, Bonventre JV, Sabbisetti VS, Waikar SS, Mifflin TE, et al.; CKD Biomarkers Consortium. Urinary Biomarkers and Risk of ESRD in the Atherosclerosis Risk in Communities Study. Clin J Am Soc Nephrol. 2015 Nov;10(11):1956-63.
15 Jotwani V, Scherzer R, Abraham A, et al. Association of urine a1-microglobulin with kidney function decline and mortality in HIVinfected women. Clin J Am Soc Nephrol. 2015 Jan 7;10(1):63-73.

16 Jotwani V, Scherzer R, Estrella MM, Jacobson LP, Witt MD, Palella FJ Jr, et al. HIV Infection, Tenofovir, and Urine $\alpha 1$-Microglobulin: A Cross-sectional Analysis in the Multicenter AIDS Cohort Study. Am J Kidney Dis. 2016 Oct;68(4):571-81.

17 Ascher SB, Scherzer R, Estrella M, Shlipak M, $\mathrm{Ng}$ D, Palella F, et al. Associations of urine biomarkers with kidney function decline in HIV-infected and uninfected men. Am J Nephrol.Sept.,2019,DOI:10.1159/000502898.
18 Ix JH, Katz R, Bansal N, Foster M, Weiner DE, Tracy R, et al. Urine Fibrosis Markers and Risk of Allograft Failure in Kidney Transplant Recipients: A Case-Cohort Ancillary Study of the FAVORIT Trial. Am J Kidney Dis. 2017 Mar;69(3):410-9.

19 Ascher SB, Scherzer R, Estrella MM, Zhang WR, Muiru AN, Jotwani V, et al. Association of Urinary Biomarkers of Kidney Injury with Estimated GFR Decline in HIV-Infected Individuals following Tenofovir Disoproxil Fumarate Initiation. Clin J Am Soc Nephrol. 2018 Sep;13(9):1321-9. 